PROCEEDINGS OF THE

AMERICAN MATHEMATICAL SOCIETY

Volume 128, Number 3, Pages 691-700

S 0002-9939(99)05616-6

Article electronically published on October 20, 1999

\title{
REFINEMENT OF STRONG MULTIPLICITY ONE FOR AUTOMORPHIC REPRESENTATIONS OF $G L(n)$
}

\author{
C. S. RAJAN
}

(Communicated by Dennis A. Hejhal)

\begin{abstract}
We state a qualitative form of strong multiplicity one for $G L_{1}$. We derive refinements of strong multiplicity one for automorphic representations arising from Eisenstein series associated to a Borel subgroup on $G L(n)$, and for the cuspidal representations on $G L(n)$ induced from idele class characters of cyclic extensions of prime degree. These results are in accordance with a conjecture of D. Ramakrishnan. We also show that Ramakrishnan's conjecture follows from a weak form of Ramanujan's conjecture. We state a conjecture concerning the structural aspects of refinements of strong multiplicity one for a pair of general automorphic representations.
\end{abstract}

\section{INTRODUCTION}

Let $K$ be a global field, and let $\mathbf{A}_{K}$ denote the ring of adeles of $K$. Suppose $\pi_{1}$ and $\pi_{2}$ are automorphic representations of $G L_{n}\left(\mathbf{A}_{K}\right)$. Define

$$
\operatorname{SM}\left(\pi_{1}, \pi_{2}\right)=\left\{v \in M_{K} \mid \pi_{1, v} \simeq \pi_{2, v}\right\},
$$

where $M_{K}$ denotes the set of places of $K$, and $\pi_{1, v}$ (resp. $\pi_{2, v}$ ) denotes the local components of $\pi_{1}$ (resp. $\pi_{2}$ ) at the place $v$ of $K$. The question of strong multiplicity one is the following:

Under what hypothesis on $S M\left(\pi_{1}, \pi_{2}\right)$ can one conclude $\pi_{1} \simeq \pi_{2}$ ?

If the complement of $S M\left(\pi_{1}, \pi_{2}\right)$ is finite and $\pi_{1}, \pi_{2}$ are unitary cuspidal automorphic representations, then it is known by the strong multiplicity one theorem of Jacquet, Piatetski-Shapiro and Shalika [JS], [JPSh], that $\pi_{1} \simeq \pi_{2}$. In view of the applications of strong multiplicity one results to base change, and other functorial questions concerning automorphic representations, we consider in this paper refinements and the structural aspects of strong multiplicity one. By the structural aspect, we mean the relationship between $\pi_{1}$ and $\pi_{2}$, assuming that the complement of $S M\left(\pi_{1}, \pi_{2}\right)$ in $M_{K}$ is of positive density.

In [DR2, page 442] D. Ramakrishnan considered the case when the complement in $M_{K}$ of $S M\left(\pi_{1}, \pi_{2}\right)$ is no longer finite, and made the following conjecture:

Conjecture 1 (D. Ramakrishnan). Let $\pi_{1}, \pi_{2}$ be unitary cuspidal automorphic representations of $G L_{n}\left(\mathbf{A}_{K}\right)$. Let $T$ be a set of places of $K$ of Dirichlet density strictly less than $1 / 2 n^{2}$. Suppose that for $v \notin T, \pi_{1, v} \simeq \pi_{2, v}$. Then $\pi_{1} \simeq \pi_{2}$.

Received by the editors April 28, 1998.

1991 Mathematics Subject Classification. Primary 11F70; Secondary 11F12, 22E55.

(C)1999 American Mathematical Society 
In [DR1], D. Ramakrishnan showed that the conjecture is true when $n=2$. In [R2] the analogous conjecture for $l$-adic representations was established. In this paper, we present some more evidence towards this conjecture when $n>2$. We show that if $\pi_{1}$ and $\pi_{2}$ are automorphically induced representations of $G L_{n}\left(\mathbf{A}_{K}\right)$ from idele class characters, or if they arise from the construction of Eisenstein series corresponding to the Borel in $G L_{n}$, then the conjecture is true.

We first state a qualitative form of the strong multiplicity one theorem for $G L_{1}$. The theorem for $G L_{n}$ follows from applying the qualitative strong multiplicity one result for $G L_{1}$, and reducing to the case when the characters involved are Dirichlet characters, which can then be reduced to a question on finite groups. We also show that Ramakrishnan's conjecture follows from knowing the Ramanujan conjecture, and this presents another proof of our main theorem.

\section{2. $G L(1)$}

We recall the notion of upper density. The upper density $u d(P)$ of a set $P$ of primes of $K$ is defined to be the ratio

$$
u d(P)=\limsup _{x \rightarrow \infty} \#\left\{v \in \Sigma_{K} \mid N v \leq x, v \in P\right\} / \#\left\{v \in \Sigma_{K} \mid N v \leq x\right\},
$$

where $N v$, the norm of $v$, is the cardinality of the finite set $\mathcal{O}_{K} / \mathfrak{p}_{v}, \mathcal{O}_{K}$ is the ring of integers of $K$, and $\mathfrak{p}_{v}$ is the prime ideal of $\mathcal{O}_{K}$ corresponding to the finite place $v$ of $K$. A set $P$ of primes is said to have a density $d(P)$, if the limit exists as $x \rightarrow \infty$ of the ratio $\#\left\{v \in \Sigma_{K} \mid N v \leq x, v \in P\right\} / \#\left\{v \in \Sigma_{K} \mid N v \leq x\right\}$, and is equal to $d(P)$. A set $P$ of primes is said to have a Dirichlet density $D(P)$ if

$$
\sum_{v \in P} N v^{-s}=-D(P) \log (s-1)+\theta_{P}(s),
$$

where $\theta_{P}(s)$ extends to a holomorphic function for $\operatorname{Re}(s) \geq 1$. It is known that if a set of primes has Dirichlet density $D(P)$, then it has density $d(P)=D(P)$ in the above sense. By a Dirichlet character we mean an idele class character of finite order. We now state a theorem, which can be considered as a qualitative form of the strong multiplicity one theorem for $G L(1)$, and is essentially due to Hecke.

Theorem 1. Let $\theta_{1}$ and $\theta_{2}$ be two idele class quasi-characters on $K$. Suppose that the set of primes $v$ of $K$ for which $\theta_{1, v}=\theta_{2, v}$ is of positive upper density. Then $\theta_{1}=\chi \theta_{2}$ for some Dirichlet character $\chi$ on $K$. In particular the set of primes at which the local components of $\theta_{1}$ and $\theta_{2}$ coincide has a density.

Proof. Let $\theta=\theta_{1} \theta_{2}^{-1}$. Suppose $\theta$ is a unitary idele class character, which is surjective restricted to the set $J_{K}^{0}$ of idele classes of norm 1 . It is shown in [L, Chapter VIII] that the set of values of $\theta_{v}\left(\pi_{v}\right)$, where $v$ runs over the set of primes where $\theta$ is unramified, is uniformly distributed on the circle. This result is essentially due to Hecke. Together with the following lemma, this shows that $\theta$ is of finite order, and hence the theorem follows.

Lemma 2. Let $\theta$ be a character of the form $\chi||^{\sigma+i t}$, for some Dirichlet character $\chi$ and some real number $t \neq 0$. Let $a \in \mathbf{C}$. Then there exist at most two rational primes $p_{1}, p_{2}$ with $\left(p_{1} p_{2}, N\right)=1$, such that for some prime $v_{i} \mid p_{i}, \theta_{v_{i}}=a, i=1,2$.

Proof of the lemma. Suppose not. Let $p_{3} \neq p_{1}, p_{2}$ be a rational prime satisfying the conclusion of the lemma. Suppose $\chi$ is of order $k$. We have $\left|\pi_{v_{i}}\right|^{k \sigma+k i t}=$ $a^{k}, v_{i} \mid p_{i}, i=1,2,3$. From $\left|\pi_{v_{1}} / \pi_{v_{2}}\right|^{k \sigma+k i t}=1$, we obtain $\sigma=0$. Using the ratios 
of these equations, we deduce that $\left(\log \left|\pi_{v_{1}} / \pi_{v_{2}}\right|\right) /\left(\log \left|\pi_{v_{2}} / \pi_{v_{3}}\right|\right) \in \mathbf{Q}$, which implies that the primes $p_{1}, p_{2}, p_{3}$ are multiplicatively dependent, which is not possible.

Remark. There does not seem to be any reference to the above strong multiplicity one result in the literature. In [Li], the theorem is used in the form, that if the set of primes of $K$, where the local component of $\theta$ is principal, is of positive density, then $\theta$ is of finite order. The above theorem also implies Ramakrishnan's conjecture is true for $n=1$, that if the local components of $\theta_{1}$ and $\theta_{2}$ agree on a set of primes of density $>1 / 2$, then $\theta_{1}=\theta_{2}$. We would like to point out that the corollary says more than Ramakrishnan's conjecture for $G L_{1}$, and can be considered as a qualitative form of strong multiplicity one for $G L_{1}$. Thus if the local components of two idele class characters coincide on a set of places of $K$ of positive upper density, then the set of finite primes where they coincide has a well defined density and is precisely the set of finite primes which decompose completely in some cyclic extension of $K$. It would be interesting to know the arithmetical structure of the set of places where the local components of two automorphic representations coincide. For example, providing an arithmetical characterisation to the set of primes $v$, for which $\theta_{v}=1$, for any idele class character, or more generally to the sets of primes occurring in the Lang-Trotter conjectures, would provide a partial answer to the problem of finding a Galois theoretical interpretation for $C_{K}$.

The strong multiplicity one result for $G L_{1}$ follows also from the finiteness of class numbers if we assume only that the archimedean components of $\theta_{1}$ and $\theta_{2}$ coincide. When the grossencharacter is algebraic of type $A_{0}$ in the sense of Weil, it is possible to give a direct proof, which confirms our theorem. A proof of the following proposition can be found in [Ha, page 95], and we include the proof of this 'folklore' result for the sake of exposition. We would like to thank D. Prasad for pointing out the following proposition.

Proposition 3. Let $K$ be a Galois extension of $\mathbf{Q}$, and let $w$ be an unramified prime of degree 1 over $\mathbf{Q}$. Suppose $\theta$ is an algebraic character of type $A_{0}$, and $\theta_{w}=1$. Then $\theta$ is of finite order.

Proof. A character $\theta$ is said to be of type $A_{0}$ if $\theta$ restricted to the archimedean components $\prod_{\lambda \in M_{\infty}} K_{\lambda}^{*}$ is of the form

$$
\theta\left(\left(\alpha_{\lambda}\right)_{\lambda \in M_{\infty}}\right)=\prod_{\lambda \in M_{\infty}} \alpha_{\lambda}^{r_{\lambda}} \bar{\alpha}_{\lambda}^{s_{\lambda}}
$$

for some integers $r_{\lambda}$ and $s_{\lambda}$, and where $\lambda$ runs over the collection of archimedean places $M_{\infty}$ of $K$. At each finite prime $v$ of $K, \mathcal{O}_{v}^{*}$ is a compact, profinite group. Hence the image $\theta_{v}\left(\mathcal{O}_{v}\right)^{*}$ is a finite subgroup of $\mathbf{C}^{*}$. By considering a suitable power of $\theta$, we can assume that $\theta$ is unramified at all finite primes of $K$. From the finiteness of class number, we can find an element $\alpha_{w}$ of $K^{*}$, which generates $\mathfrak{p}_{w}^{m} \subset \mathcal{O}_{K}$ for some integer $m>0 . \alpha_{w}$ is necessarily a unit at all finite primes $w^{\prime} \neq w$ of $K$. Since $\theta$ is unramified at all the finite primes and $\theta_{w}=1$, it follows from the properties of $\alpha_{w}$ that $\theta\left(\left(\alpha_{w_{\lambda}}\right)_{\lambda \in M_{\infty}}\right)=1$. Since $w$ is unramified of degree 1 over $\mathbf{Q}^{*}$, the conjugates of $\alpha_{w}$ are multiplicatively independent. Hence we have from the equality that $\prod_{\lambda \in M_{\infty}} \alpha_{w_{\lambda}}^{r_{\lambda}} \bar{\alpha}_{w_{\lambda}}^{s_{\lambda}}=1$, that $r_{\lambda}=s_{\lambda}=0$ for all archimedean places $\lambda$, and so the character is trivial on the archimedean places. By the finiteness of class number of $K$, it follows that $\theta$ is of finite order. 
Remark. It can be observed that the proof indicates by the classical Siegel-Walfisz theorem that for a character of type $\mathcal{A}_{0}$, the following is to be expected:

$$
\#\left\{N v<x \mid \theta_{v}=1\right\}=O(x \exp (-c \sqrt{\log x})) \text { as } x \rightarrow \infty,
$$

for some positive constant $c$. In fact a result of the above type can be established for any Hecke character of infinite order [R1].

\section{3. $G L(n)$}

We will now examine some of the consequences of the multiplicity one theorem for $G L_{1}$, towards strong multiplicity one results for certain classes of automorphic representations on $G L_{n}$. For these we recall some results concerning the structure of automorphic representations on $G=G L_{n}$ ([La2]).

Let $B$ denote the Borel subgroup of $G L_{n}$ consisting of upper triangular matrices, let $U$ denote the unipotent subgroup of $B$ consisting of upper triangular matrices with the diagonal entries being 1 , and let $T \simeq G L_{1}^{n}$ denote the subgroup of $B$ consisting of diagonal matrices. Let $W:=N(T) / Z(T)$ denote the Weyl group of $G L_{n}$. By a parabolic subgroup $P$ we will always mean a standard parabolic subgroup with Levi decomposition $P=M N, T \subset M, N \subset U$.

Let $\sigma$ be an irreducible cuspidal automorphic representation of $M\left(\mathbf{A}_{K}\right)$. It is known by the theory of Eisenstein series ([La1], [La2]), that any irreducible automorphic representation $\pi$ of $G L_{n}\left(\mathbf{A}_{K}\right)$ is an irreducible subquotient of $I_{P}(\sigma):=$ $\operatorname{Ind}_{P\left(\mathbf{A}_{K}\right)}^{G L_{n}\left(\mathbf{A}_{K}\right)}(\sigma)$, where $\sigma$ is considered as a representation of $P\left(\mathbf{A}_{K}\right)$ by extending it trivially on the subgroup $U\left(\mathbf{A}_{K}\right)$, and the induction denotes unitary induction. Further if $\pi$ is an irreducible subquotient of two such induced representations $I_{P_{1}}\left(\sigma_{1}\right)$ and $I_{P_{2}}\left(\sigma_{2}\right)$, then it is known that there is an element $w \in W$, such that $M_{1}^{w}=M_{2}$ and $\sigma_{1}^{w^{-1}} \simeq \sigma_{2}$. In this case we will call such data $\left(P_{1}, \sigma_{1}\right)$ and $\left(P_{2}, \sigma_{2}\right)$ associate data, and the corresponding parabolic subgroups $P_{1}$ and $P_{2}$ associate parabolic subgroups. Given a class of associate parabolic subgroups $P \in \mathcal{P}$, let $\mathcal{A}_{\mathcal{P}}$ denote the collection of irreducible automorphic representations of $G L_{n}\left(\mathbf{A}_{K}\right)$ which occur as an irreducible subquotient of $I_{P}(\sigma)$, for some $P \in \mathcal{P}$ and $\sigma$ an irreducible cuspidal automorphic representation of $M\left(\mathbf{A}_{K}\right)$. Thus the collection of irreducible automorphic representations of $G L_{n}\left(\mathbf{A}_{K}\right)$ can be written as a disjoint union of $\mathcal{A}_{\mathcal{P}}$, indexed by the equivalence classes of associate parabolics.

Specialising to the case when the associate class consists of only the Borel subgroup $B$, we see that an element of $\mathcal{A}_{\mathcal{B}}$ is an irreducible subquotient of the representation $I_{P}(\chi)$, where $\chi=\left(\chi_{1}, \cdots, \chi_{n}\right)$ is a character of $T\left(\mathbf{A}_{K}\right) / T(K) \simeq$ $\left(G L_{1}\left(\mathbf{A}_{K}\right) / K^{*}\right)^{n}$ and where $\chi_{i}, i=1, \cdots, n$, are idele class characters of $K$.

Example. The characters $\chi$ which contribute to the residual discrete spectrum of $G L_{n}\left(\mathbf{A}_{K}\right)$ are known by the theorem of Moeglin and Waldspurger ([MoW]). Let $\xi, \chi_{0}$ be idele class characters of $K$ with $\chi_{0}^{n}=\xi$. Let $\chi$ be the character sending $\left(t_{1}, \cdots, t_{n}\right) \in T\left(\mathbf{A}_{K}\right) / T(K)$ to $\left(\chi_{0}\left|t_{1}\right|^{(n-1) / 2}, \cdots, \chi_{0}\left|t_{n}\right|^{(1-n) / 2}\right)$. Let $J(\chi)$ denote the unique irreducible quotient of $I_{B}(\chi)$. Then the $J(\chi)$ are precisely the automorphic representations of $G L_{n}\left(\mathbf{A}_{K}\right)$ with central character $\xi$, occurring in the discrete spectrum and $\mathcal{A}_{B}$.

Suppose $\pi$ is an irreducible automorphic representation of $G L_{n}\left(\mathbf{A}_{K}\right)$. Then $\pi$ can be written as a restricted tensor product $\pi={ }^{\prime} \otimes{ }^{\prime} \pi_{v}$, of irreducible representations of the local groups $G L_{n}\left(K_{v}\right)$, where for all but finitely many primes $v$ of $K, \pi_{v}$ is 
an unramified representation of $G L_{n}\left(K_{v}\right)$. Let $\pi$ be an irreducible subquotient of $I_{P}(\sigma)$. For almost all primes $v$ of $K, \pi_{v}$ is the unique irreducible subquotient of Ind $_{P\left(K_{v}\right)}^{G L_{n}\left(K_{v}\right)}\left(\chi_{v}\right)$ which contains the trivial representation of $G L_{n}\left(\mathcal{O}_{v}\right)([\mathrm{La} 2])$, where $\mathcal{O}_{v}$ denotes the ring of integers of the local field $K_{v}$. These representations are the spherical principal series indexed by the unramified characters of $T\left(K_{v}\right)$. It is known by the theory of spherical principal series ([Ca]) that given an unramified character $\chi_{v}=\left(\chi_{1, v}, \cdots, \chi_{n, v}\right)$ of $T\left(K_{v}\right)$, then there is a unique irreducible subquotient of $\operatorname{Ind}_{B\left(K_{v}\right)}^{G L_{n}\left(K_{v}\right)}(\chi)$, denoted by $\pi\left(\chi_{v}\right)$, which contains the trivial representation of $G L_{n}\left(\mathcal{O}_{v}\right)$. Morever $\pi\left(\chi_{v}\right) \simeq \pi\left(\chi_{v}^{\prime}\right)$ if and only if the characters $\chi_{v}$ and $\chi_{v}^{\prime}$ are associate, i.e., the corresponding sets of characters are equal, $\left\{\chi_{1, v}, \cdots, \chi_{n, v}\right\}=$ $\left\{\chi_{1, v}^{\prime}, \cdots, \chi_{n, v}^{\prime}\right\}$.

Let $L$ be a cyclic extension of $K$ of degree a prime number $r$. Let $\sigma$ be the non-trivial element of the Galois group of $L$ over $K$. Corresponding to an idele class character $\theta$ on $L$, which is not fixed by $\sigma$, Kazhdan has constructed an irreducible, cuspidal automorphic representations $I_{L}^{K}(\theta)$ on $G L_{r}\left(\mathbf{A}_{K}\right)([\mathrm{AC}])$. For $r=2$, these representations were constructed by Labesse and Langlands. These cuspidal representions have the property that under the base change morphism $B C_{L / K}$ from automorphic representations on $G L_{r}\left(\mathbf{A}_{K}\right)$ to automorphic representations on $G L_{r}\left(\mathbf{A}_{L}\right)$, we have

$$
B C_{L / K}\left(I_{L}^{K}(\theta)\right)=\pi\left(\theta, \theta^{\sigma}, \cdots, \theta^{\sigma^{r-1}}\right) .
$$

Let $\mathcal{C}_{K}$ denote the collection of irreducible, automorphic representations on $G L_{r}\left(\mathbf{A}_{K}\right)$, which are either of the type constructed by Kazhdan or belong to $\mathcal{A}_{B}$.

We obtain then a strong form of multiplicity one theorem for these representations. We start with a lemma on finite groups, which implies in particular that Ramakrishnan's conjecture is true for Artin type representations of Galois groups into $G L(n, \mathbf{C})$. Although this lemma is well known, and was one of the principal motivations for Ramakrishnan's conjecture, we include this for the sake of exposition.

Lemma 4. Let $G$ be a finite group and let $\rho_{1}, \rho_{2}$ be inequivalent representations of $G$ into $G L(n, \mathbf{C})$. Then

$$
\#\left\{g \in G \mid \operatorname{Tr}\left(\rho_{1}(g)\right)=\operatorname{Tr}\left(\rho_{1}(g)\right)\right\} \leq\left(1-1 / 2 n^{2}\right)|G|
$$

If morever, $\rho_{1}$ and $\rho_{2}$ are sums of one dimensional characters, then

$$
\#\left\{g \in G \mid \operatorname{Tr}\left(\rho_{1}(g)\right)=\operatorname{Tr}\left(\rho_{1}(g)\right)\right\} \leq(1-1 / 2 n)|G| .
$$

Proof. If $\rho_{1} \neq \rho_{2}$, then by orthogonality relations,

$$
\frac{1}{|G|} \sum_{g \in G}\left|\operatorname{Tr}\left(\rho_{1}(g)\right)-\operatorname{Tr}\left(\rho_{2}(g)\right)\right|^{2} \geq 2 .
$$

Let $\Psi=\left\{g \in G \mid \operatorname{Tr}\left(\rho_{1}(g)\right)=\operatorname{Tr}\left(\rho_{2}(g)\right)\right\}$. Since these are representations of finite groups, the character value at an element $g$ in $G$ is a sum of $n$ roots of unity. Hence

$$
\begin{aligned}
\sum_{g \in G}\left|\operatorname{Tr}\left(\rho_{1}(g)\right)-\operatorname{Tr}\left(\rho_{2}(g)\right)\right|^{2} & =\sum_{g \in G-\Psi}\left|\operatorname{Tr}\left(\rho_{1}(g)\right)-\operatorname{Tr}\left(\rho_{2}(g)\right)\right|^{2} \\
& \leq(|G|-|\Psi|) 4 n^{2}
\end{aligned}
$$

Hence $|\Psi| \leq\left(1-1 / 2 n^{2}\right)|G|$. 
In the second case, we have from the orthogonality relations that

$$
\frac{1}{|G|} \sum_{g \in G}\left|\operatorname{Tr}\left(\rho_{1}(g)\right)-\operatorname{Tr}\left(\rho_{2}(g)\right)\right|^{2} \geq 2 n .
$$

Arguing as above then yields the result.

Theorem 5. i) Let $\pi_{1}$ and $\pi_{2}$ be representations belonging to $\mathcal{A}_{B}$. Suppose that the local components of $\pi_{1}$ and $\pi_{2}$ coincide on a set of places of upper density strictly greater than $1-1 / 2 n$. Then $\pi_{1} \simeq \pi_{2}$.

ii) Let $r$ be a prime and let $\pi_{1}$ and $\pi_{2}$ be representations belonging to $\mathcal{C}_{K}$ of $G L_{r}\left(\mathbf{A}_{K}\right)$. Suppose that the local components of $\pi_{1}$ and $\pi_{2}$ coincide on a set of places of upper density strictly greater than $1-1 / 2 r^{2}$. Then $\pi_{1} \simeq \pi_{2}$.

In particular if $\pi_{1}$ and $\pi_{2}$ are either non-cuspidal or dihedral cuspidal automorphic representations of $G L\left(2, \mathbf{A}_{K}\right)$, and if the local components of $\pi_{1}$ and $\pi_{2}$ coincide on a set of places of upper density strictly greater than $7 / 8$, then $\pi_{1} \simeq \pi_{2}$.

Proof. Let $\pi_{1}$ (resp. $\left.\pi_{2}\right)$ be an irreducible constituent of $\operatorname{Ind}_{B}^{G}(\mu)\left(\operatorname{resp} \cdot \operatorname{Ind}_{B}^{G}(\nu)\right)$, where $\mu=\left(\mu_{1}, \cdots, \mu_{n}\right)$ (resp. $\nu=\left(\nu_{1}, \cdots, \nu_{n}\right)$ ). At an unramified prime $v$ for $\pi_{1}$ and $\pi_{2}$, the local components of $\pi_{1}$ (resp. $\pi_{2}$ ) are the spherical principal series $\pi\left(\mu_{1, v}, \cdots, \mu_{n, v}\right)$ (resp. $\left.\pi\left(\nu_{1, v}, \cdots, \nu_{n, v}\right)\right)$. By the characterisation of spherical principal series $([\mathrm{Ca}])$, we have that $\left\{\mu_{1, v}, \cdots, \mu_{n, v}\right\}=\left\{\nu_{1, v}, \cdots, \nu_{n, v}\right\}$, at a set of places of $K$ of positive upper density. In fact by Theorem 1 and our hypothesis, the set of unramified places $v$ where $\left\{\mu_{1, v}, \cdots, \mu_{n, v}\right\}=\left\{\nu_{1, v}, \cdots, \nu_{n, v}\right\}$ has positive density.

Let us define two idele class characters to be equivalent if they are twists of each other by a Dirichlet character. Let us also assume that the two sets of characters are not identical. After a possible rearrangement let $\nu_{1}, \cdots, \nu_{k}$ be the subset of characters of $\left\{\nu_{1}, \cdots, \nu_{n}\right\}$ which are equivalent to $\mu_{1}$. Under the hypothesis of the theorem, Theorem 1 implies that we have, up to a rearrangement, $\nu_{1}=\chi_{1} \mu_{1}, \cdots, \nu_{n}=\chi_{n} \mu_{n}$, for some Dirichlet characters $\chi_{i}, i=1, \cdots, n$, on $K$. It follows that the characters $\mu_{1}, \cdots, \mu_{k}, \nu_{1}, \cdots, \nu_{k}$ are precisely the characters which are equivalent to $\mu_{1}$. Thus we have decomposed the characters into two sets of characters $\left\{\mu_{1}, \cdots, \mu_{k}, \nu_{1}, \cdots, \nu_{k}\right\}$ and $\left\{\mu_{k+1}, \cdots, \mu_{n}, \nu_{k+1}, \cdots, \nu_{n}\right\}$, with the property that any two characters in the first decomposition are equivalent to each other, and no character from the first decomposition is equivalent to any of the characters from the second decomposition. By appealing to Theorem 1, we see that the density of places at which the local components of $\left\{\mu_{1}, \cdots, \mu_{n}\right\}$ and $\left\{\nu_{1}, \cdots, \nu_{n}\right\}$ coincide is less than the upper density of places at which the local components of $\left\{\mu_{1}, \cdots, \mu_{k}\right\}$ and $\left\{\nu_{1}, \cdots, \nu_{k}\right\}$ coincide. Thus we have reduced to the case when all the characters that we consider are equivalent to one another. Cancelling $\mu_{1}$ from both sides, we have reduced to the case where we have to bound the density of primes $v$, at which $\left\{\eta_{1, v} \cdots, \eta_{n, v}\right\}=\left\{\chi_{1, v}, \cdots, \chi_{n, v}\right\}$ are equal, and where $\eta_{i}, \chi_{j}(1 \leq i, j \leq n)$ are now arbitrary Dirichlet characters on $K$.

By class field theory, we can assume that the characters $\chi_{i}, \eta_{i}, 1 \leq i \leq n$, are characters of some finite Galois group $G$ over $K$. We can consider the representations $\chi, \eta: G \rightarrow G L(n, \mathbf{C})$, defined respectively by $\chi=\sum_{i=1}^{n} \chi_{i}$ and $\eta=\sum_{i=1}^{n} \eta_{i}$. The equality of the local components at a place $v$ implies in particular the equality of the traces of $\chi$ and $\eta$ at $v$, i.e., $\operatorname{Tr}\left(\chi\left(\sigma_{v}\right)\right)=\operatorname{Tr}\left(\eta\left(\sigma_{v}\right)\right)$, where $\sigma_{v}$ is the Frobenius at $v$. The first part of the theorem then follows from the above lemma. 
To prove the second half of the theorem, we use the base change map $B C_{L / K}$ ([AC]), from automorphic representations of $G L_{r}\left(\mathbf{A}_{K}\right)$ to automorphic representations of $G L_{r}\left(\mathbf{A}_{L}\right)$. Let $\delta$ be the density of the set $S_{L}$ of places of $L$, where the local components of $B C_{L / K}\left(\pi_{1}\right)$ and $B C_{L / K}\left(\pi_{2}\right)$ coincide. Then the density of primes of $K$, which split completely in $L$ and for which the local components of $\pi_{1}$ and $\pi_{2}$ coincide, is $\delta /[L: K]$. Since the representations $\pi_{1}$ and $\pi_{2}$ belong to $\mathcal{C}_{K}$, it follows that $B C_{L / K}\left(\pi_{1}\right)$ and $B C_{L / K}\left(\pi_{2}\right)$ are in $\mathcal{A}_{B}$, corresponding to $G L(n)$ over the field $L$. From the first part of the theorem, we obtain that $\delta \leq 1-1 / 2 r$. Hence it follows that the set $S_{K}$ of places $v$ of $K$, at which $\pi_{1, v} \simeq \pi_{2, v}$, consists of at most the places of $K$ which are inert in $L$, together with those places of $K$ which split completely in $L$ and belong to $S_{L}$. Hence the density of $S_{K}$ is at most $(1-1 / r)+1 / r(1-1 / 2 r)=1-1 / 2 r^{2}$.

The last statement about $G L(2)$ follows from the characterisation of non-cuspidal representations of $G L(2)$ given by Theorem 10 of $([\mathrm{JL}])$.

Remark. This theorem should be compared with the refinement of the strong multiplicity one theorem by D. Ramakrishnan ([DR1]), which states that if the local components of two irreducible, unitary, cuspidal automorphic representations on $G L_{2}$ agree on a set of primes of density at least $7 / 8$, then the representations coincide.

In analogy with $G L_{1}$ and motivated by the analogous results established in [R2] for $l$-adic representations, we conjecture the following, which clarifies the structural aspects of strong multiplicity one, and is stronger than Conjecture 1. We refer to [La3, page 210] for the following notions. Let $H$ be a reductive group over $K$. Let $\mathcal{L}$ denote the conjectural Langlands group possessing the property that to an 'admissible' homomorphism $\phi$ of $\mathcal{L}$ into the Langlands dual ${ }^{L} H$ of $H$, there is 'associated' a finite equivalence class of automorphic representations of $H\left(\mathbf{A}_{K}\right)$ and conversely. This association is such that at all but finitely many places $v$ of $K$, the local parameter $\phi_{v}$, which can be considered as a representation of the local DeligneWeil group $W\left(K_{v}\right)$ into ${ }^{L} H$, should correspond via the conjectural local Langlands correspondence to the local component $\pi_{v}$ of $\pi$, where $\pi$ is an element of this class. For almost all places $v$ of $K, \pi_{v}$ will be an unramified spherical representation of $H\left(K_{v}\right)$, and there is a well defined Frobenius conjugacy class $\phi_{v}\left(F_{v}\right)$ inside $G L_{n}(\mathbf{C})$. Denote by $a_{v}(\pi)$ the trace of this conjugacy class at such a prime $v$. Suppose $\pi$ is an isobaric automorphic representation of $G L_{n}\left(\mathbf{A}_{K}\right)$ such that the local components $\pi_{v}$ are tempered. The image $H(\pi):=\phi_{\pi}(\mathcal{L})$ will be a reductive subgroup of $G L_{n}(\mathbf{C})$. Consider now two irreducible automorphic representations $\pi_{1}$ and $\pi_{2}$ of $G L_{n}\left(\mathbf{A}_{K}\right)$, such that the local components are tempered. Let

$$
\operatorname{SMT}\left(\pi_{1}, \pi_{2}\right)=\left\{v \in \Sigma_{K}-S \mid a_{v}\left(\pi_{1}\right)=a_{v}\left(\pi_{2}\right)\right\},
$$

where $S$ is a finite set of places containing the ramified places of $\pi_{1}$ and $\pi_{2}$. In analogy with the results of [R2], we can make the following conjecture:

Conjecture 2. a) Suppose that the connected components of $H\left(\pi_{1}\right)$ and $H\left(\pi_{2}\right)$ are not conjugate inside $G L_{n}(\mathbf{C})$. Then $\operatorname{SMT}\left(\pi_{1}, \pi_{2}\right)$ is of density zero.

b) Suppose that $H\left(\pi_{1}\right)$ is connected and acts irreducibly on the natural representation $\mathbf{C}^{n}$. Suppose that $S M T\left(\pi_{1}, \pi_{2}\right)$ has positive upper density. Then there exists an idele class character $\chi$ of finite order such that for all but finitely many places $v$ of $K, \pi_{2, v} \simeq\left(\pi_{1} \otimes \chi\right)_{v}$. 
In particular for $G L_{2}$, the above conjecture says the following: suppose $\pi_{1}$ is a cuspidal non-dihedral automorphic representation and $\pi_{2}$ is not a cuspidal nondihedral automorphic representation of $G L_{2}\left(\mathbf{A}_{K}\right)$. Then $S M T\left(\pi_{1}, \pi_{2}\right)$ is of density zero. Morever suppose $\pi_{1}, \pi_{2}$ are irreducible, cuspidal, non-dihedral representations of $G L_{2}\left(\mathbf{A}_{K}\right)$ such that the local components of $\pi_{1}$ and $\pi_{2}$ coincide for a positive density of places of $K$. Then there exists a Dirichlet character $\chi$ of $K$, such that $\pi_{2} \simeq \pi_{1} \otimes \chi$.

The proof of Theorem 5 can be modified to give a proof that the above conjecture implies Ramakrishnan's conjecture.

Remark. Note that in Theorem 5 what we actually give is an upper bound for $\operatorname{SMT}\left(\pi_{1}, \pi_{2}\right)$, where $\pi_{1}$ and $\pi_{2}$ are distinct automorphic representations belonging to $\mathcal{A}_{B}$. However one can ask the question for an upper bound for $S M\left(\pi_{1}, \pi_{2}\right)$, i.e., the equality of the local components up to isomorphism. Now $\pi_{1, v}=\pi\left(\mu_{1}, \cdots, \mu_{n}\right)$ $\simeq \pi\left(\nu_{1}, \cdots, \nu_{n}\right)=\pi_{2, v}$ is equivalent to equality of the sets of characters

$$
\left\{\mu_{1, v}, \cdots, \mu_{n, v}\right\}=\left\{\nu_{1, v}, \cdots, \nu_{n, v}\right\} .
$$

In this regard we make the following curious combinatorial conjecture, which we can prove when all the characters involved are quadratic characters, which is the extreme case. Note that the jumps in density occur at powers of 2 .

Conjecture 3. Let $\pi_{1}=\pi\left(\mu_{1}, \cdots, \mu_{n}\right)$ and $\pi_{2}=\pi\left(\nu_{1}, \cdots, \nu_{n}\right)$ be two irreducible, automorphic representations on $G L_{n}\left(\mathbf{A}_{K}\right)$, belonging to the set $\mathcal{A}_{B}$, for some idele class characters $\mu_{1}, \cdots, \mu_{n}, \nu_{1}, \cdots, \nu_{n}$ on $K$. If the local components of $\pi_{1}$ and $\pi_{2}$ agree on a set of primes of density strictly greater than $\delta(n):=\left(2^{d(n)}-1\right) / 2^{d(n)}$, then $\left\{\mu_{1}, \cdots, \mu_{n}\right\}=\left\{\nu_{1}, \cdots, \nu_{n}\right\}$, where $d(n)=\left[\log _{2}(2 n)\right]$. In particular $\pi\left(\mu_{1}, \cdots, \mu_{n}\right)$ $\simeq \pi\left(\nu_{1}, \cdots, \nu_{n}\right)$.

In this revised version we give a brief outline, skipping some of the details, of a different proof of Theorem 5, which mimics the proof of Lemma 4, given for finite groups. This result was independently observed by D. Ramakrishnan. Let us say that an automorphic representation $\pi$ of $G L_{n}\left(\mathbf{A}_{K}\right)$ satisfies the weak Ramanujan conjecture [DR3], if for $v \notin S$, we have

$$
\text { Weak Ramanujan conjecture: }\left|a_{v}(\pi)\right| \leq n \quad \forall v \notin S .
$$

Note that if $\pi$ is a unitary representation belonging to $\mathcal{A}_{\mathcal{B}}$, then $\pi$ satisfies the weak Ramanujan conjecture.

Theorem 6. Suppose $\pi_{1}$ and $\pi_{2}$ are irreducible, unitary, cuspidal automorphic representations of $G L_{n}\left(\mathbf{A}_{K}\right)$, unramified outside a finite set of places $S$ of $K$ and satisfying the weak Ramanujan conjecture. Then Conjecture 1 is true.

Proof. Let $\tilde{\pi}$ denote the contragredient representation of $\pi$, and for two automorphic representations $\pi$ and $\eta$, let $L(s, \pi \times \eta)$ denote the Rankin-Selberg $L$-function. Let $S$ be a finite set of places containing the ramified places of $\pi_{1}$ and $\pi_{2}$. Consider the following Dirichlet series:

$$
L(s)=\frac{L_{S}\left(s, \pi_{1} \times \tilde{\pi}_{1}\right) L_{S}\left(s, \pi_{2} \times \tilde{\pi}_{2}\right)}{L_{S}\left(s, \pi_{1} \times \tilde{\pi}_{2}\right) L_{s}\left(s, \pi_{2} \times \tilde{\pi}_{1}\right)} .
$$

It follows from properties of Rankin-Selberg convolutions proved by Jacquet, Piatetski-Shapiro, Shalika and Shahidi [JPSh], [Sh] that $L(s)$ has a pole of order 2 at $s=1$, under the assumption that $\pi_{1}$ and $\pi_{2}$ are distinct. In fact, one has 
the following:

$$
\operatorname{Lim}_{s \rightarrow 1^{+}}-\frac{\log L(s)}{\log (s-1)}=2
$$

Writing $L(s)$ in terms of the Euler product expansion, using the estimate on the eigenvalues given by Luo, Rudnick and Sarnak [LRS], and standard analytic number theory estimates, it can be checked that

$$
\log L(s)=\sum_{v \notin S}\left|a_{v}\left(\pi_{1}\right)-a_{v}\left(\pi_{2}\right)\right|^{2} N v^{-s}+O(1) \text { as } s \rightarrow 1^{+} .
$$

Let $T$ be a set of places $v$ with Dirchlet density $D(T)$ containing $S$, such that for $v \notin T, a_{v}\left(\pi_{1}\right)=a_{v}\left(\pi_{2}\right)$. By the hypothesis of our theorem that $\pi_{1}$ and $\pi_{2}$ satisfy the weak Ramanujan conjecture, it follows that

$$
\log L(s) \leq \sum_{v \in T} 4 n^{2} N v^{-s}+O(1) \quad \text { as } s \rightarrow 1^{+} .
$$

By definition of the Dirichlet density for a set of primes, we have

$$
D(T)=\operatorname{Lim}_{s \rightarrow 1^{+}}-\frac{\sum_{v \in T} N v^{-s}}{\log (s-1)} \geq \operatorname{Lim}_{s \rightarrow 1^{+}}-\frac{\log L(s)}{4 n^{2} \log (s-1)}=\frac{1}{2 n^{2}},
$$

and that provides a proof of the theorem.

Remark. The same proof can be modified to prove part (i) of Theorem 5 .

\section{ACKNOWLEDGEMENT}

I am indebted to M. Ram Murty and D. Ramakrishnan for many discussions and for their suggestions. I also thank my colleagues at CICMA and Caltech for their warm hospitality and support during my stay at these places, in which period part of this work was done. I thank the referee for his suggestions which improved the exposition of this paper.

\section{REFERENCES}

[AC] J. Arthur and L. Clozel, Simple algebras, base change and the advanced theory of the trace formula, Annals of Maths Studies 120 (1989), Princeton Univ. Press. MR 90m:22041

[Ca] P. Cartier, Representation theory of p-adic groups, A survey in Automorphic forms, representations and $L$-functions, Proc. Symp. Pure Math., vol. 33, Part 1, Amer. Math. Soc., Providence, R.I., 1977, pp. 111-155. MR 81e:22029

[GL] P. Gérardin and J. P. Labesse, The solution of a base change problem for GL(2), in Automorphic forms, representations and $L$-functions, Proc. Symp. Pure Math., vol. 33, Part 2, Amer. Math. Soc., Providence, R.I., 1977, pp. 115-133. MR 82e:10047

[Ha] G. Harder, Some results on the Eisenstein cohomology of arithmetic subgroups of $G L_{n}$, in Cohomology of arithmetic groups and automorphic forms, eds., J. -P. Labesse and J. Schwermer, Lect. Notes in Math. 1447, Springer-Verlag, 1990. MR 91j:11040

[He] E. Hecke, Eine neue Art von Zetafunktionen und ihre Beziehungen zur Verteilung der Primzahlen, Zweite Mitteilung, Mathematische Werke 14 249-289.

[JL] H. Jacquet and R. Langlands, Automorphic forms on GL(2), SLN 114, New York, 1970. MR 53:5481

[JS] H. Jacquet and J. Shalika, Euler products and the classification of automorphic forms, I and II, Amer. J. Math. 103 (1981), 499-558 and 777-815. MR 82m:10050a; MR 82m:10050b

[JPSh] H. Jacquet, I. Piatetski-Shapiro and J. Shalika, Rankin-Selberg convolutions, Amer. J. Math. 105 (1983), 367-464. MR 85g:11044

[L] S. Lang, Algebraic Number Theory, Grad. Texts in Math., Springer-Verlag, New York, 1986. MR 44:181 
[La1] R. Langlands, On the functional equations satisfied by Eisenstein series, Lecture Notes in Math., vol. 544, Springer, New York, 1976. MR 58:28319

[La2] R. Langlands, On the notion of an automorphic representation, in Automorphic forms, representations and $L$-functions, Proc. Symp. Pure Math., vol. 33, Part 1, Amer. Math. Soc., Providence, R.I., 1977, pp. 203-207. MR 81m:10055

[La3] R. P. Langlands, Automorphic representations, Shimura varieties and motives. Ein märchen, Proc. Symp. Pure Math., 33, vol. 2, Amer. Math. Soc., 1979, 205-246. MR 83f: 12010

[Li] W.-C. W. Li, On converse theorems for $G L(2)$ and $G L(1)$, Amer. J. Math., 103, 1981, 851-885. MR 83b:22024

[LRS] W. Luo, Z. Rudnick and P. Sarnak, On the generalized Ramanujan conjecture for $G L(n)$, preprint.

[MoW] C. Möeglin and J. -L Waldspurger, Le spectre résiduel de GL(n), Ann. Sci. École Norm. Sup. (4) 22 (1989), no. 4, 605-674. MR 91b:22028

[MR] M. R. Murty and C. S. Rajan, Stronger multiplicity one theorems for forms of general type on $G L_{2}$, in Analytic Number Theory, Volume 2, Proceedings of a conference in honour of Heine Halberstam, edited by B. Berndt, H. Diamond, and A. Hilderbrand, Birkhauser, Boston, 1996, pp. 669- 683. MR 97h:11047

[R1] C. S. Rajan, Distribution of values of Hecke characters of infinite order, Acta Arith., 85 (1998), no. 3, 279-291. MR 99e:11142

[R2] C. S. Rajan, On strong multiplicity one for l-adic representations, Internat. Math. Res. Notices 1998, no. 3, 161-172. MR 99c:11064

[DR1] D. Ramakrishnan, Appendix: A refinement of the strong multiplicity one theorem for GL(2), Invent. Math., 116, 1994, 645-649. MR 95h:11050b

[DR2] D. Ramakrishnan, Pure motives and autmorphic forms, Motives, Proc. Symp. Pure Math., 55, vol. 2, Amer. Math. Soc., 1994, 411-446. MR 94m:11134

[DR3] D. Ramakrishnan, On the coefficients of cusp forms, Math. Res. Letters 4 (1997), 295307. MR 98e: 11064

[Sh] F. Shahidi, On certain L-functions, Amer. J. Math. 103 (1981), 297-355. MR 82i:10030

School of Mathematics, Tata Institute of Fundamental Research, Homi Bhabha ROAD, BOMBAY - 400 005, INDIA

E-mail address: rajan@math.tifr.res.in 